\title{
A Multivariate Analysis of Forecast Disagreement: \\ Confronting Models of Disagreement with Survey Data
}

\author{
Jonas Dovern
}

Heidelberg University

\section{SEM Conference - Paris}

Juli 22-24, 2015

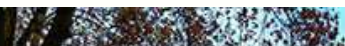

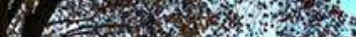

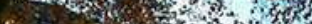

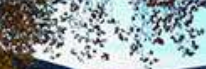

5

A

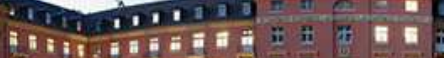

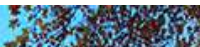

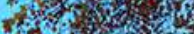

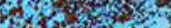
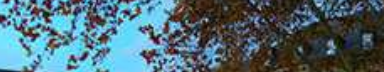

s.

4. 


\section{Motivation}

Disagreement about Inflation Outlook (EA) as Measured from SPF Data (2015q2)
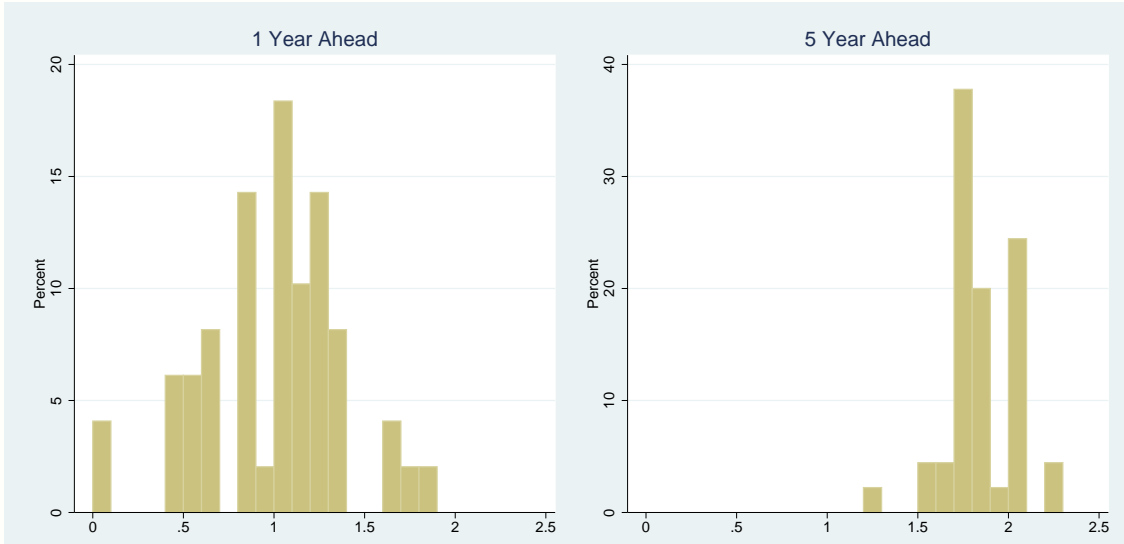


\section{Motivation}

- Disagreement about future values of macroeconomic variables is high

- So far, only univariate disagreement has been analyzed empirically

- Models of heterogeneous expectation formation are calibrated/evaluated based on information about univariate/marginal disagreement 


\section{Motivation}

- Disagreement about future values of macroeconomic variables is high

- So far, only univariate disagreement has been analyzed empirically

- Models of heterogeneous expectation formation are calibrated/evaluated based on information about univariate/marginal disagreement

- What properties does multivariate forecast disagreement have? 


\section{Motivation}

- Disagreement about future values of macroeconomic variables is high

- So far, only univariate disagreement has been analyzed empirically

- Models of heterogeneous expectation formation are calibrated/evaluated based on information about univariate/marginal disagreement

- What properties does multivariate forecast disagreement have?

- Which implications does this have for theoretical models of heterogenous expectation formation? 


\section{Motivation}

A Stylized Scatter of Forecasts

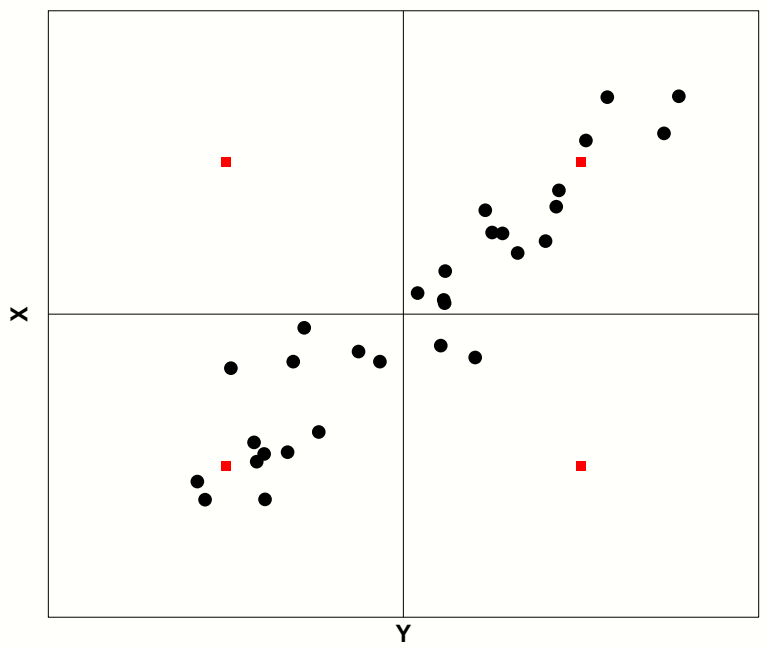




\section{Contribution of Paper}

- Documentation of multivariate disagreement in macroeconomic expectations

- In particular, two new stylized facts:

- Forecasters disagree about the relationship between different macroeconomic variables

- Different forecasters tend to have different levels of disagreement

- Discussion of implications for models of heterogeneous expectation formation:

- Along which dimensions do models fail?

- Modifications of models to fit observed multivariate disagreement better

- Suggestion how multivariate aspects can be taken into account during estimation of models

- What I do not:

- Abstracting from behavioral issues (e.g., incentives or interaction between forecasters)

- Not yet considering option of model uncertainty 


\section{Contribution of Paper}

- Documentation of multivariate disagreement in macroeconomic expectations

- In particular, two new stylized facts:

- Forecasters disagree about the relationship between different macroeconomic variables

- Different forecasters tend to have different levels of disagreement

- Discussion of implications for models of heterogeneous expectation formation:

- Along which dimensions do models fail?

- Modifications of models to fit observed multivariate disagreement better

- Suggestion how multivariate aspects can be taken into account during estimation of models

- What I do not:

- Abstracting from behavioral issues (e. g., incentives or interaction between forecasters)

- Not yet considering option of model uncertainty 


\section{Agenda}

(2) Contribution of Paper

\section{(3) Related Literature}

(4) Measurement of Disagreement

(5) Data and Stylized Facts

(6) Implications for Models of Disagreement

(7) Conclusion 


\section{Related Literature}

- Empirical analyses of forecast disagreement (Mankiw et al., 2003; Lahiri and Sheng, 2008; Patton and Timmermann, 2010; Rich and Tracy, 2010; Coibion and Gorodnichenko, 2012; Andrade and Le Bihan, 2013; Andrade et al., 2014; Dovern et al., 2012)

- Empirical studies with multivariate aspects (Banternghansa and McCracken, 2009; Dräger and Lamla, 2015)

- Reduced-form models of heterogenous expectations:

- Noisy information models (Coibion and Gorodnichenko, 2012; Andrade and Le Bihan, 2013; Andrade et al., 2014)

- Models with agent-specific priors (Lahiri and Sheng, 2008; Patton and Timmermann, 2010)

- Sticky information models (Mankiw et al., 2003; Coibion and Gorodnichenko, 2012)

- DSGE models with heterogenous expectation formation:

- Models with constant levels of disagreement (Nimark, 2008; Maćkowiak and Wiederholt, 2009; Lorenzoni, 2009)

- Sticky information DSGE models (Mankiw and Reis, 2002)

- “Man-bites-dog” model (Nimark, 2014) 


\section{Related Literature}

- Empirical analyses of forecast disagreement (Mankiw et al., 2003; Lahiri and Sheng, 2008; Patton and Timmermann, 2010; Rich and Tracy, 2010; Coibion and Gorodnichenko, 2012; Andrade and Le Bihan, 2013; Andrade et al., 2014; Dovern et al., 2012)

- Empirical studies with multivariate aspects (Banternghansa and McCracken, 2009; Dräger and Lamla, 2015)

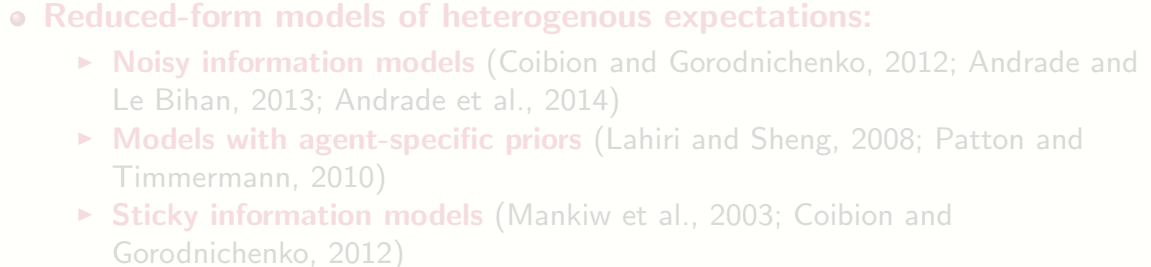

- DSGE models with heterogenous expectation formation:

- Models with constant levels of disagreement (Nimark, 2008; Maćkowiak and Wiederholt, 2009; Lorenzoni, 2009)

- Sticky information DSGE models (Mankiw and Reis, 2002)

- “Man-bites-dog" model (Nimark, 2014) 


\section{Agenda}

\section{(1) Motivation}

(2) Contribution of Paper

(3) Related Literature

(4) Measurement of Disagreement

(5) Data and Stylized Facts

(6) Implications for Models of Disagreement

(7) Conclusion 


\section{How to Measure Multivariate Disagreement}

- Measures proposed by Banternghansa and McCracken (2009)

- Basic inputs: vector-valued forecasts $\left(y_{i, t+h \mid t}\right)$ and their (cross-sectional) covariance

$$
S_{t+h \mid t}=N_{t+h \mid t}^{-1} \sum_{i=1}^{N_{t+h \mid t}}\left(y_{i, t+h \mid t}-\bar{y}_{t+h \mid t}\right)\left(y_{i, t+h \mid t}-\bar{y}_{t+h \mid t}\right)^{\prime}
$$

- Overall/Multivariate disagreement (absolute)

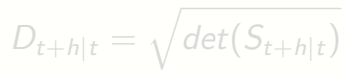

- Individual disagreement (relative):

Intuition/Examples 


\section{How to Measure Multivariate Disagreement}

- Measures proposed by Banternghansa and McCracken (2009)

- Basic inputs: vector-valued forecasts $\left(y_{i, t+h \mid t}\right)$ and their (cross-sectional) covariance

$$
S_{t+h \mid t}=N_{t+h \mid t}^{-1} \sum_{i=1}^{N_{t+h \mid t}}\left(y_{i, t+h \mid t}-\bar{y}_{t+h \mid t}\right)\left(y_{i, t+h \mid t}-\bar{y}_{t+h \mid t}\right)^{\prime}
$$

- Overall/Multivariate disagreement (absolute):

$$
D_{t+h \mid t}=\sqrt{\operatorname{det}\left(S_{t+h \mid t}\right)}
$$




\section{How to Measure Multivariate Disagreement}

- Measures proposed by Banternghansa and McCracken (2009)

- Basic inputs: vector-valued forecasts $\left(y_{i, t+h \mid t}\right)$ and their (cross-sectional) covariance

$$
S_{t+h \mid t}=N_{t+h \mid t}^{-1} \sum_{i=1}^{N_{t+h \mid t}}\left(y_{i, t+h \mid t}-\bar{y}_{t+h \mid t}\right)\left(y_{i, t+h \mid t}-\bar{y}_{t+h \mid t}\right)^{\prime}
$$

- Overall/Multivariate disagreement (absolute):

$$
D_{t+h \mid t}=\sqrt{\operatorname{det}\left(S_{t+h \mid t}\right)}
$$

- Individual disagreement (relative):

$$
d_{i, t+h \mid t}=\sqrt{\left(y_{i, t+h \mid t}-\bar{y}_{t+h \mid t}\right)^{\prime} S_{t+h \mid t}^{-1}\left(y_{i, t+h \mid t}-\bar{y}_{t+h \mid t}\right)}
$$




\section{Agenda}

(3) Related Literature

(4) Measurement of Disagreement

(5) Data and Stylized Facts

(6) Implications for Models of Disagreement

(7) Conclusion 


\section{Data}

- Survey of Professional Forecasters for Euro area:

- Forecasts from research institutes, banks, and large corporations

- Sample: 1999q1-2015q1

- 1-year-ahead forecasts $(h=4)$ and long-term forecasts $(h=20)$

- Forecasts for growth, inflation and the unemployment rate

- Survey of Professional Forecasters for United States:

- Longer sample: 1981q3-2014q4

- 1-quarter-ahead forecasts $(h=1)$ and 1-year-ahead forecasts $(h=4)$

- Robustness check with additional forecasts for short- and long-term interest rates and industrial production

- Consensus Economics survey data for twelve advanced economies:

- Fixed-event forecasts (annual averages)

- Concentrate on forecasts with $h=13$

- Sample: 1990m1-2015m4 


\section{Multivariate Disagreement $\left(D_{t+h \mid t}\right)$ over Time}

(a) US-SPF

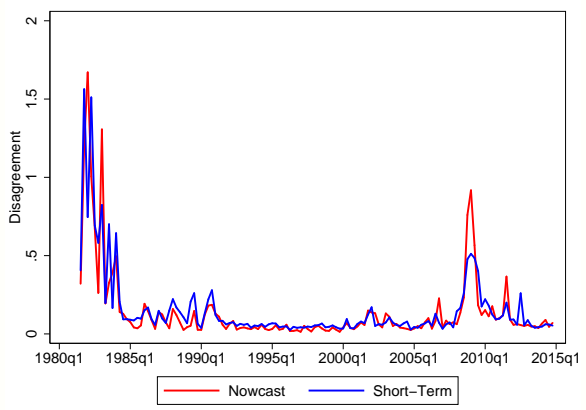

(b) EA-SPF

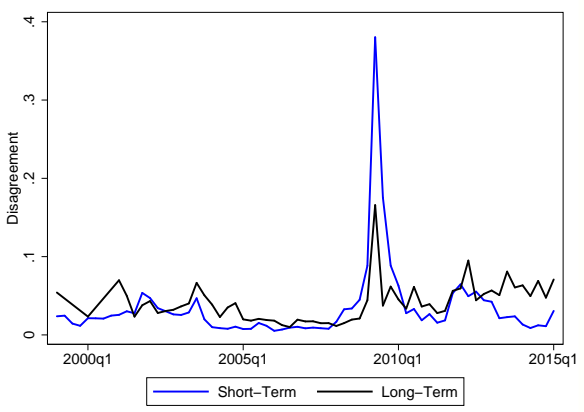




\section{... a Closer Look at Individual Components}

United States: Short-Term $(h=4)$
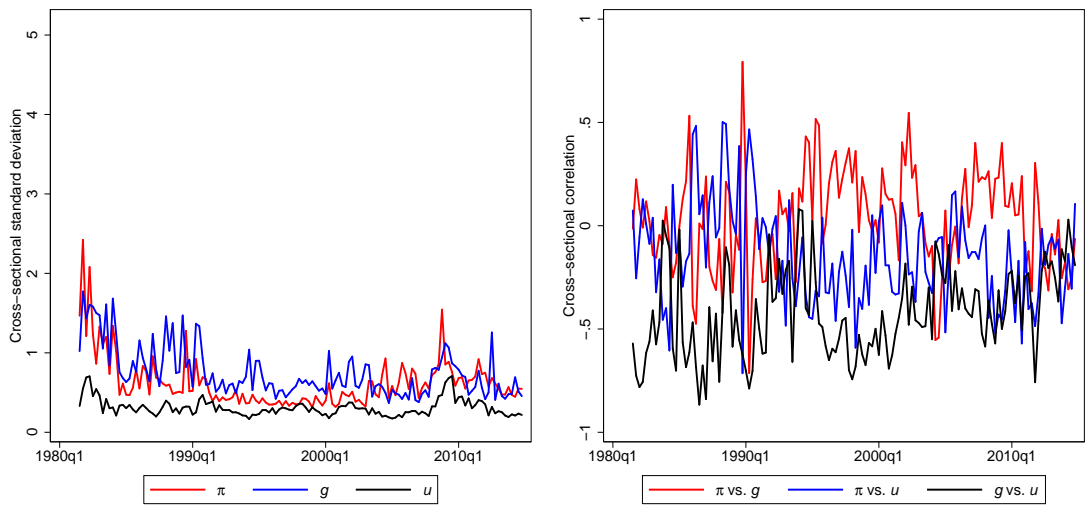


\section{... a Closer Look at Individual Components}

\section{Unconditional Averages}

\begin{tabular}{lrrrrr}
\hline & \multicolumn{2}{c}{ Euro area } & & \multicolumn{2}{c}{ US } \\
& $h=4$ & $h=20$ & & $h=1$ & $h=4$ \\
\cline { 2 - 3 } \cline { 5 - 6 }$s d(g)$ & 0.36 & 0.27 & & 1.07 & 0.75 \\
$s d(\pi)$ & 0.27 & 0.22 & & 0.88 & 0.62 \\
$s d(u)$ & 0.31 & 0.65 & & 0.12 & 0.31 \\
$\operatorname{Corr}(g, \pi)$ & 0.16 & 0.10 & & 0.02 & 0.03 \\
$\operatorname{Corr}(g, u)$ & -0.25 & -0.17 & -0.17 & -0.42 \\
$\operatorname{Corr}(\pi, u)$ & -0.10 & -0.02 & -0.07 & -0.14 \\
\hline
\end{tabular}

Notes: The values shown are based on the averages for each element of $S_{t+h \mid t}$ over the full sample. These averages are computed as $\bar{s}_{i j, h}=\sum_{t=1}^{T} s_{t+h \mid t}^{i j}$, where $s_{t+h \mid t}^{i j}$ denotes the element of $S_{t+h \mid t}$ corresponding to the $\mathrm{i}^{\text {th }}$ row and the $\mathrm{j}^{\text {th }}$ column. $\operatorname{sd}(\bullet)$ is computed as the square root of the main diagonal elements. $\operatorname{Corr}(\bullet, \bullet)$ is computed as the correlations that correspond to the off-diagonal elements. 


\section{Assessment of Rank Persistence (based on $d_{i, t+h \mid t}$ )}

Visualizing Persistence ...
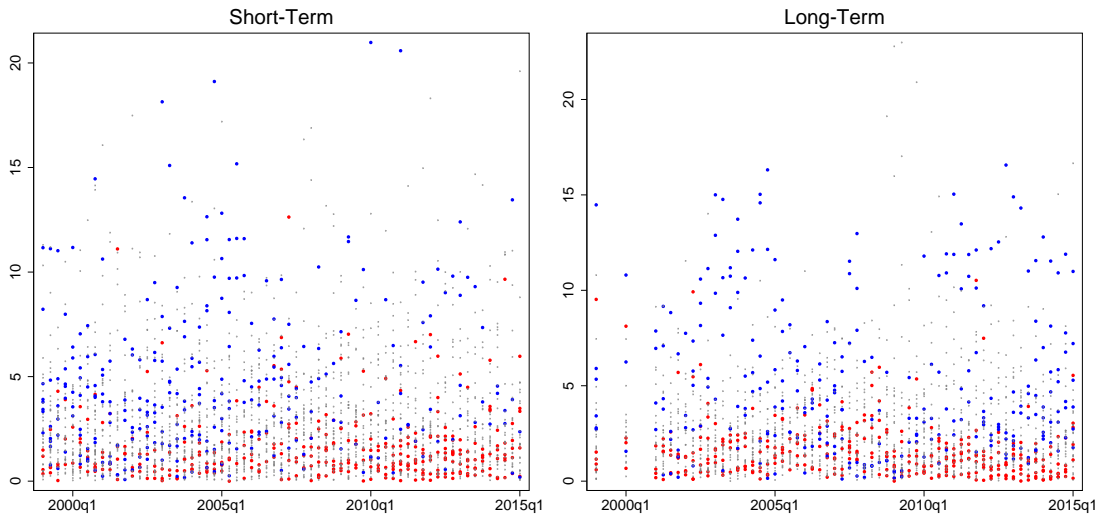


\section{Assessment of Rank Persistence}

Measurement

- Kendall's coefficient of concordance: Formal assessment of rank persistence

- $\mathrm{W}=0$ : No persistence

- $\mathrm{W}=1$ : Perfectly stable ranking 


\section{Assessment of Rank Persistence}

\section{EA-SPF/US-SPF}

\begin{tabular}{lccccc}
\hline & \multicolumn{2}{c}{ Euro area } & & \multicolumn{2}{c}{ US } \\
& $h=4$ & $h=20$ & & $h=1$ & $h=4$ \\
\cline { 2 - 3 } \cline { 5 - 6 } Since 1981q3 & - & - & & 0.37 & 0.43 \\
Since 1999q1 & 0.14 & 0.21 & & 0.25 & 0.34 \\
1999q1-2008q2 & 0.16 & 0.31 & & 0.27 & 0.39 \\
Since 2008q3 & 0.22 & 0.37 & & 0.33 & 0.45 \\
\hline
\end{tabular}

For comparison (since 1999q1):

Point forecasts

$\begin{array}{lllll}g & 0.13 & 0.30 & 0.18 & 0.28 \\ \pi & 0.19 & 0.44 & 0.13 & 0.39 \\ u & 0.14 & 0.27 & 0.13 & 0.28\end{array}$

Forecast uncertainty

\begin{tabular}{lllll}
$g$ & 0.56 & 0.65 & - & - \\
$\pi$ & 0.32 & 0.62 & - & - \\
$u$ & 0.54 & 0.56 & - & - \\
\hline
\end{tabular}

Notes: The table shows values of Kendall's coefficient of concordance. The statistics have been calculated by accounting for missing values as proposed in Boero et al. (2014). 


\section{Assessment of Rank Persistence}

\section{Consensus Forecast Data}

\begin{tabular}{lccccccc}
\hline & \multicolumn{3}{c}{ Full Sample } & & \multicolumn{3}{c}{ Since 1999 } \\
& $h=1$ & $h=13$ & $h=24$ & & $h=1$ & $h=13$ & $h=24$ \\
\cline { 2 - 3 } \cline { 7 - 8 } USA & 0.21 & 0.24 & 0.25 & & 0.27 & 0.22 & 0.25 \\
Japan & 0.14 & 0.26 & 0.31 & & 0.19 & 0.32 & 0.35 \\
Germany & 0.16 & 0.17 & 0.19 & & 0.22 & 0.20 & 0.20 \\
France & 0.29 & 0.29 & 0.33 & & 0.33 & 0.32 & 0.24 \\
UK & 0.30 & 0.36 & 0.42 & & 0.33 & 0.31 & 0.50 \\
Italy & 0.25 & 0.24 & 0.27 & & 0.23 & 0.19 & 0.26 \\
Canada & 0.26 & 0.22 & 0.24 & & 0.40 & 0.24 & 0.25 \\
Netherlands & 0.21 & 0.17 & 0.31 & & 0.32 & 0.27 & 0.39 \\
Norway & 0.21 & 0.22 & 0.17 & & 0.21 & 0.19 & 0.17 \\
Spain & 0.38 & 0.30 & 0.22 & 0.43 & 0.25 & 0.25 \\
Sweden & 0.26 & 0.20 & 0.30 & 0.25 & 0.21 & 0.21 \\
Switzerland & 0.24 & 0.24 & 0.25 & 0.25 & 0.24 & 0.25 \\
\hline Average & 0.24 & 0.24 & 0.27 & 0.29 & 0.25 & 0.28 \\
\hline
\end{tabular}

Notes: The table shows values of Kendall's coefficient of concordance. The statistics have been calculated by accounting for missing values as proposed in Boero et al. (2014). 


\section{Agenda}

\section{(1) Motivation}

(2) Contribution of Paper

(3) Related Literature

(4) Measurement of Disagreement

(5) Data and Stylized Facts

6) Implications for Models of Disagreement 


\section{Implications for Models of Disagreement}

Andrade et al. (2014): Model Structure

- Model structure:

$$
\begin{aligned}
& y_{t}=\left(I_{k}-\Phi\right) \mu_{t}+\Phi y_{t-1}+\nu_{t}^{y} \\
& \mu_{t}=\mu_{t-1}+\nu_{t}^{\mu} \\
& y_{i t}=y_{t}+\eta_{i t}
\end{aligned}
$$

with $\nu_{t}^{y} \sim \mathcal{N}\left(0, \Sigma^{y}\right), \nu_{t}^{\mu} \sim \mathcal{N}\left(0, \Sigma^{\mu}\right)$, and $\eta_{i t} \sim \mathcal{N}\left(0, \Sigma^{\eta}\right)$

- Agents form optimal forecasts based on Kalman filtering

- Model features not in line with new stylized facts:

- No persistence of disagreement ranking

- Relatively high cross-sectional correlation of forecasts 


\section{Implications for Models of Disagreement}

Andrade et al. (2014): Effects of Introducing True Heterogeneity

- New feature: Heterogenous signal-to-noise ratios:

$$
\eta_{i t} \sim \mathcal{N}\left(0, \delta_{i} \Sigma^{\eta}\right) \quad \text { with } \quad \delta_{i} \sim \mathcal{G}\left(1, \sigma_{\delta}^{2}\right)
$$

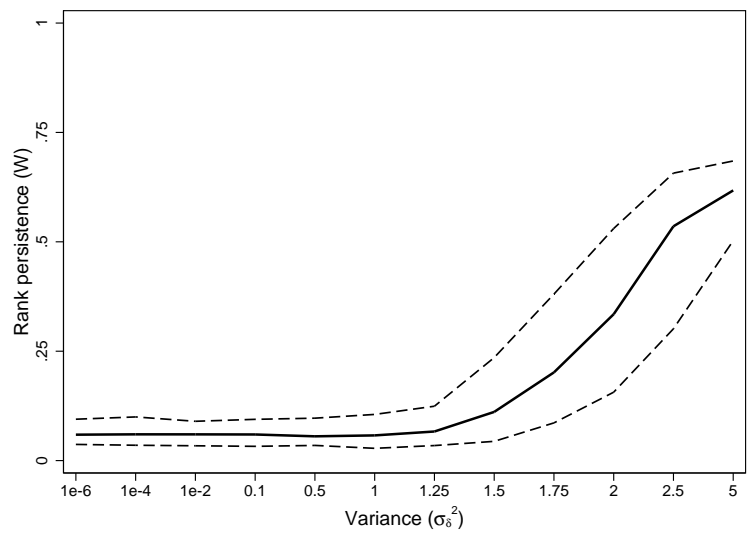




\section{Implications for Models of Disagreement}

\section{Nimark (2014): Structure of DSGE Model}

- Model structure:

- Island economy as in Lorenzoni (2009)

- Agents observe island-specific productivity $\left(a_{j, t}=a_{t}+\varepsilon_{j, t}\right)$, island-specific demand, and a sub-set of goods prices

- Additional "man-bites-dog" signal $\left(z_{t}^{a}=a_{t}+\eta_{t}\right)$ about common productivity shock

$\Rightarrow$ Probability of this signal rises with (absolute) size of productivity shock

- Fig. 1 of Nimark (2014)

- Main mechanism to generate variation in disagreement:

- Different interpretation of "man-bites-dog" signals due to different priors across agents

- Level of disagreement depends on frequency of occurrence of these signals (and dispersion of priors)

- Model is estimated and evaluated using information about univariate disagreement only 


\section{Implications for Models of Disagreement}

Nimark (2014): Implied Cross-Sectional Correlation between Different Forecasts

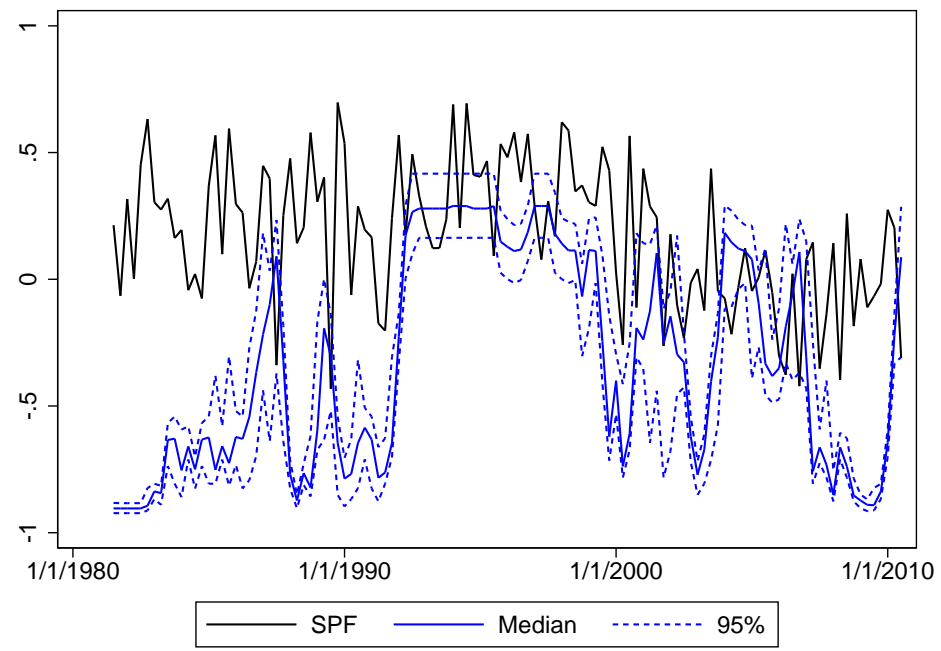




\section{Summary}

- Interesting features of multivariate disagreement that have previously been ignored in the literature

- They suggest that agents are truly heterogeneous (beyond being subject to different information flows)

- Models of heterogeneous expectation formation have neglected this dimension so far

- How can models be adapted?

- Heterogeneous signal-to-noise ratios

- Learning models? Models with different types of forecasters?

- At least, multivariate information should be used for estimation

- What can we learn in a broader context?

- Observed correlations contain information about (perceived) relevance of different types of macroeconomic shocks

- Heterogeneity of agents might not only be important in terms of the current state-vector (e.g., credit constraints) but also in terms of expectations 


\section{Background on Multivariate Measure}

$D_{t+h, t}$ as a Function of One Variance

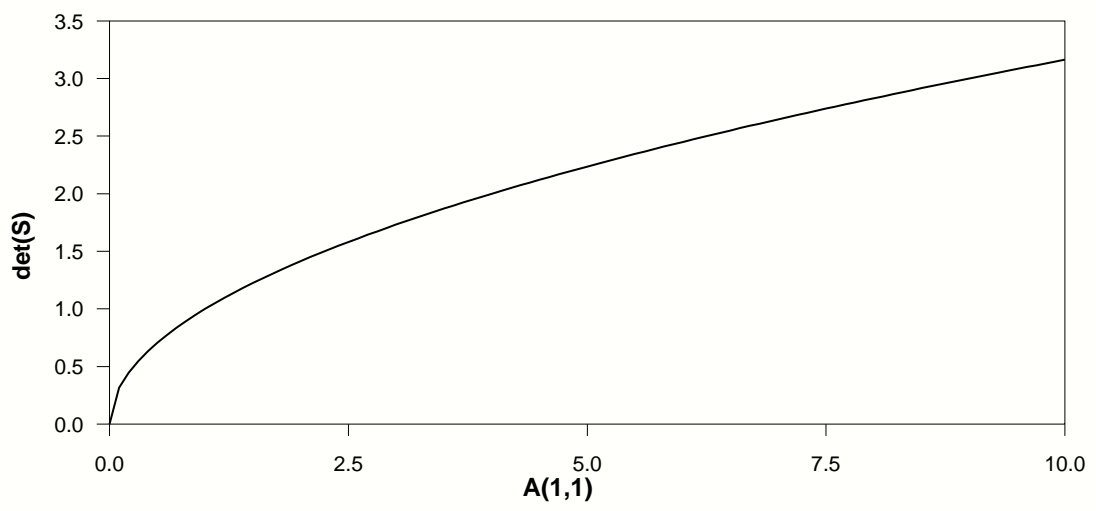




\section{Background on Multivariate Measure}

$D_{t+h, t}$ as a Function of Variances

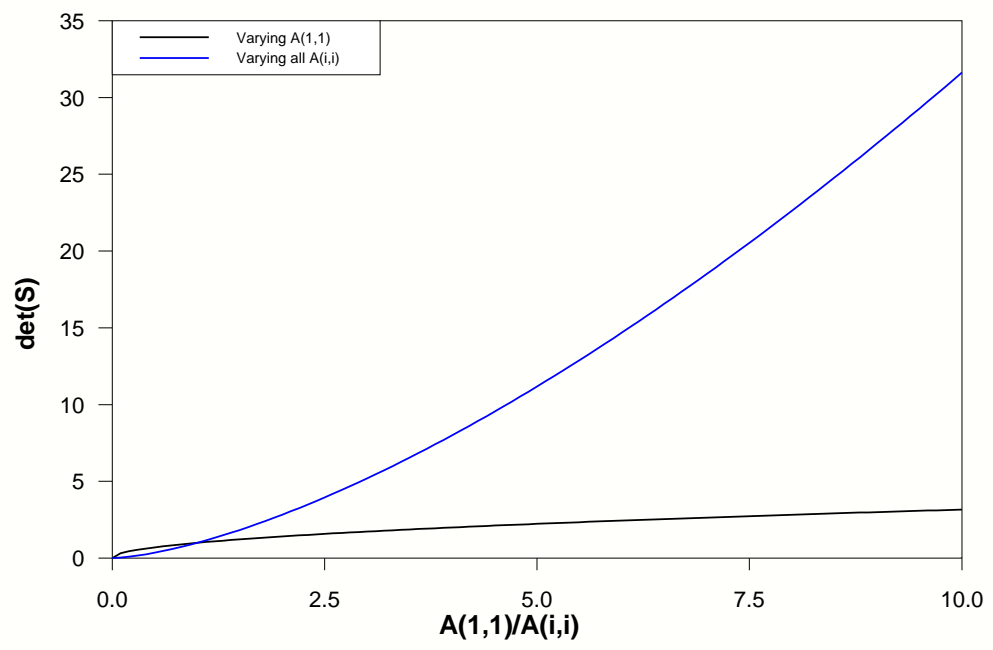




\section{Background on Multivariate Measure}

\section{$D_{t+h, t}$ as a Function of Correlations}

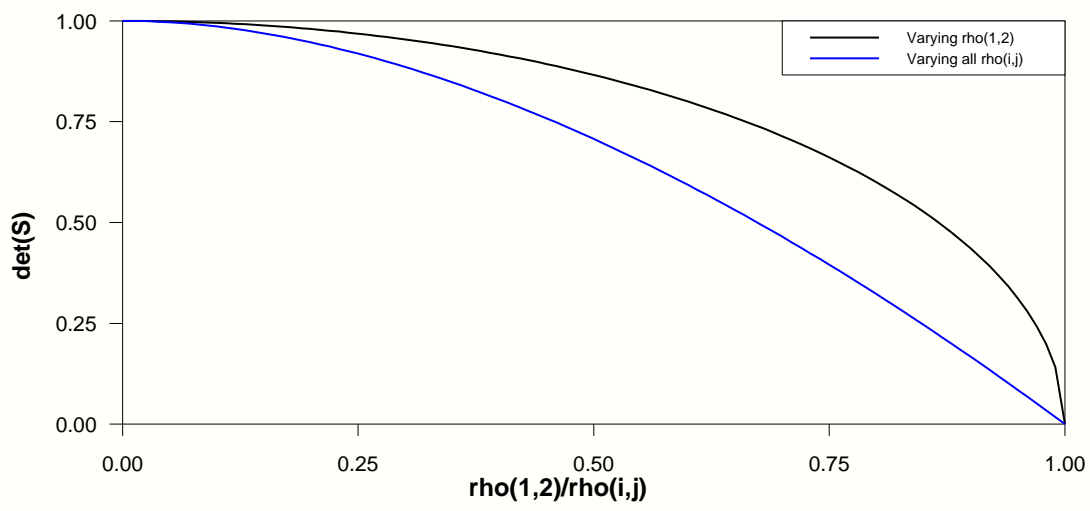

- Back 


\section{Occurrence of "Man-Bites-Dog" Signal}
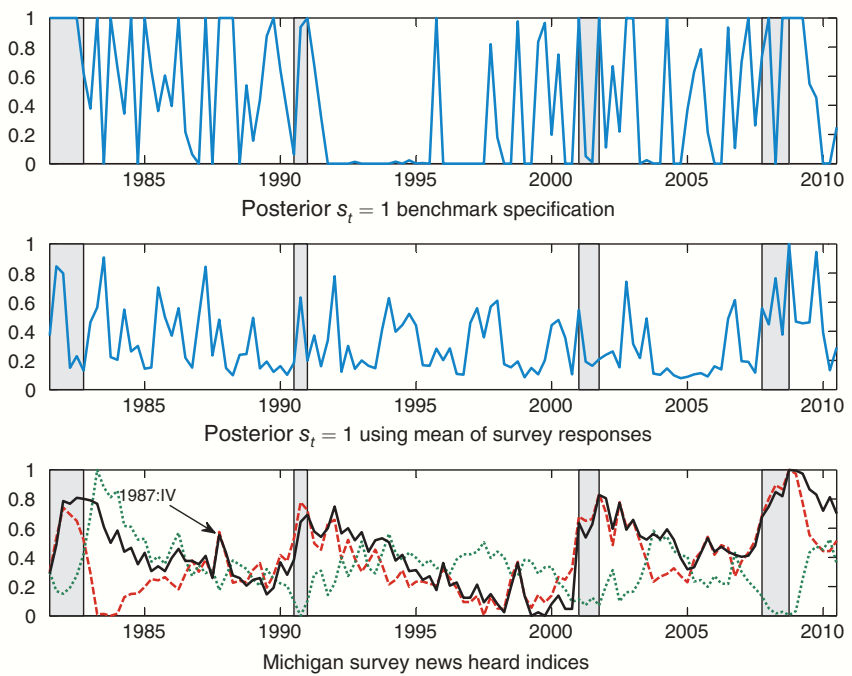


\section{Intuition behind "Man-Bites-Dog" Signal}

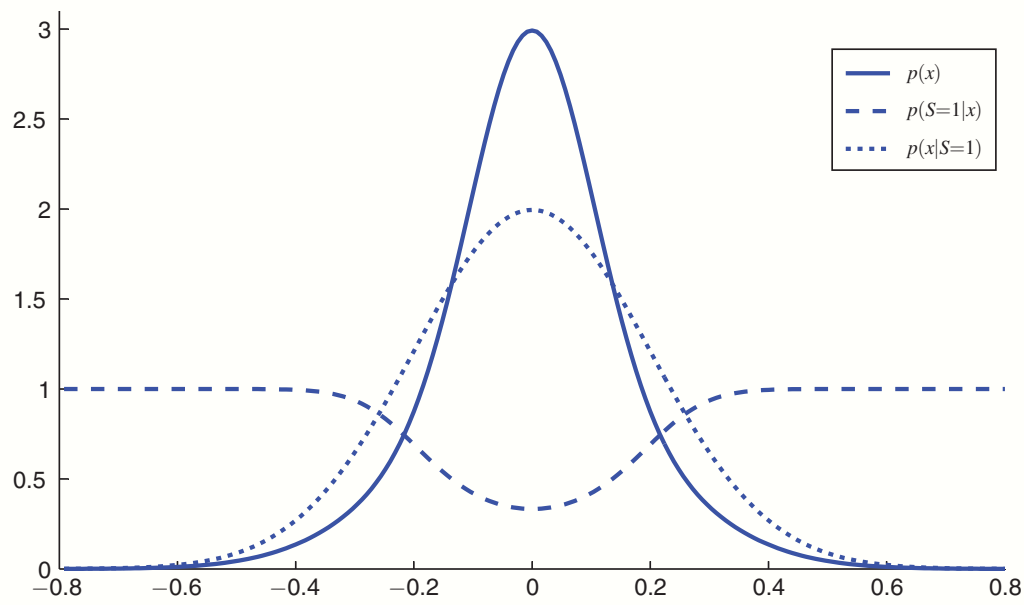

Figure 1. Unconditional Distribution of $x$, Conditional Probability of Observing the Signal $y$, and the Implied Conditional Distribution of $x$ 


\section{Implications for Models of Disagreement}

Nimark (2014): Suggestion to Take Multivariate Aspects into Account

- Modification of model to take multivariate dimension of disagreement into account

- Nimark (2014) assumes that forecasts are drawn from univariate distributions:

$$
\begin{aligned}
f_{t, \pi}^{j} & \sim \mathcal{N}\left(\int E\left[\pi_{t+1} \mid \Omega_{j, t}\right] d j, \sigma_{f \pi}^{2}\left(s^{t}\right)\right) \\
f_{t, \Delta y}^{j} & \sim \mathcal{N}\left(\int E\left[\Delta y_{t+1} \mid \Omega_{j, t}\right] d j, \sigma_{\Delta y}^{2}\left(s^{t}\right)\right)
\end{aligned}
$$

- I suggest to use a bivariate distribution with covariance matrix:

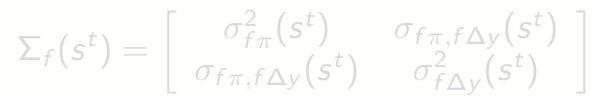

- Reflect this also in the model's LLF $\Rightarrow$ more effective use of sample

- Keep track of forecast pairs

$\checkmark$ Need not even throw away univariate forecasts 


\section{Implications for Models of Disagreement}

Nimark (2014): Suggestion to Take Multivariate Aspects into Account

- Modification of model to take multivariate dimension of disagreement into account

- Nimark (2014) assumes that forecasts are drawn from univariate distributions:

$$
\begin{aligned}
f_{t, \pi}^{j} & \sim \mathcal{N}\left(\int E\left[\pi_{t+1} \mid \Omega_{j, t}\right] d j, \sigma_{f \pi}^{2}\left(s^{t}\right)\right) \\
f_{t, \Delta y}^{j} & \sim \mathcal{N}\left(\int E\left[\Delta y_{t+1} \mid \Omega_{j, t}\right] d j, \sigma_{\Delta y}^{2}\left(s^{t}\right)\right)
\end{aligned}
$$

- I suggest to use a bivariate distribution with covariance matrix:

$$
\Sigma_{f}\left(s^{t}\right)=\left[\begin{array}{cc}
\sigma_{f \pi}^{2}\left(s^{t}\right) & \sigma_{f \pi, f \Delta y}\left(s^{t}\right) \\
\sigma_{f \pi, f \Delta y}\left(s^{t}\right) & \sigma_{f \Delta y}^{2}\left(s^{t}\right)
\end{array}\right]
$$




\section{Implications for Models of Disagreement}

Nimark (2014): Suggestion to Take Multivariate Aspects into Account

- Modification of model to take multivariate dimension of disagreement into account

- Nimark (2014) assumes that forecasts are drawn from univariate distributions:

$$
\begin{aligned}
f_{t, \pi}^{j} & \sim \mathcal{N}\left(\int E\left[\pi_{t+1} \mid \Omega_{j, t}\right] d j, \sigma_{f \pi}^{2}\left(s^{t}\right)\right) \\
f_{t, \Delta y}^{j} & \sim \mathcal{N}\left(\int E\left[\Delta y_{t+1} \mid \Omega_{j, t}\right] d j, \sigma_{\Delta y}^{2}\left(s^{t}\right)\right)
\end{aligned}
$$

- I suggest to use a bivariate distribution with covariance matrix:

$$
\Sigma_{f}\left(s^{t}\right)=\left[\begin{array}{cc}
\sigma_{f \pi}^{2}\left(s^{t}\right) & \sigma_{f \pi, f \Delta y}\left(s^{t}\right) \\
\sigma_{f \pi, f \Delta y}\left(s^{t}\right) & \sigma_{f \Delta y}^{2}\left(s^{t}\right)
\end{array}\right]
$$

- Reflect this also in the model's LLF $\Rightarrow$ more effective use of sample information:

- Keep track of forecast pairs

- Need not even throw away univariate forecasts 